\title{
Charged Thin Shell Wormholes with Variable Equations of State
}

\author{
Ali Eid ${ }^{1,2}$ \\ ${ }^{1}$ Department of Physics, College of Science, Al Imam Mohammad Ibn Saud Islamic University (IMSIU), Riyadh, KSA \\ ${ }^{2}$ Department of Astronomy, Faculty of Science, Cairo University, Giza, Egypt
}

Email address:

aeid06@yahoo.com

\section{To cite this article:}

Ali Eid. Charged Thin Shell Wormholes with Variable Equations of State. American Journal of Modern Physics.

Vol. 4, No. 3, 2015, pp. 118-124. doi: 10.11648/j.ajmp.20150403.13

\begin{abstract}
Using the Darmois-Israel formalism the dynamical analysis of Reissner Nordstrom (RN) thin shell wormholes, at the wormhole throat, are determined by considering linearized radial perturbations around static solutions. Linearized stability of thin-shell wormholes with barotropic equation of state $(\mathrm{EoS})$ and with two different EoS is derived. In the first case of variable EoS, with regular coefficients, a sequence of semi-infinite stability regions is found such that every throat in equilibrium becomes stable for a particular subsequence. In the second case, a singular EoS (in such variable EoS the coefficients is explicitly dependent on throat radius), the second derivative of the effective potential is positive definite, so linearized stability is guaranteed for every equilibrium radius.
\end{abstract}

Keywords: General Relativity, Astrophysics, Cosmology, Gravitation

\section{Introduction}

The study of the dynamics of a shell separating two backgrounds in the context of general relativity has been developed in a powerful and direct formalism since the pioneer work of Israel [1] and applied to the charged shell by Kuchar [2]. It has been applied to cosmology, mainly to inflation, Berezin et al [3], and to modeling the dynamics of the border between two regions in different states, like bubbles or between two given spaces, Sato [4]. The linearized stability analysis of spherical shells was carried out by several authors. For instance, Kim [5] analyzed Schwarzschild-de Sitter wormholes, using the cut-and-paste construction. The formalism was applied to bubbles, shells around stars and black holes, and in the construction of thin-shell wormholes (with spherical, plane and also cylindrical throats; see for example, Lobo and Crawford [6], Visser [7], Eiroa and Simeone [8]).

The barotropic EoS is a rather strong assumption usually made in studies of thin-shell dynamics. Garcia et al. [9] have argued for local thin shell wormhole solutions with variable EoS, in which surface pressure explicitly depends on both surface energy density and throat radius. More recently, a cosmological model based on a pair of linear, variable EoS has been proposed by Ponce de Leon [10]. On the other hand,
Rahaman et al. [11] and Kuhfittig [12] have constructed Morris-Thorne type wormholes supported by fluids satisfying variable EoS, in which volumetric pressure depends explicitly on volumetric energy density and spacetime coordinates [13]. At this point, it seems natural to address the linearized stability of thin shell wormholes with variable EoS. The existence of local solutions pointed out in [9] is essential for the construction of the new models.

In this work I start from Kuhffittig's treatment of energy conservation, linear EoS, and thin shell potentials to discuss the effects of variable EoS on the stability of RN wormholes.

This paper is organized as follows. In Section 2 the Darmois -Israel formalism is briefly reviewed. Match an interior RN spacetime to an exterior $\mathrm{RN}$ spacetime, the dynamical equations of thin shell wormholes are given in Section 3. The linearized stability analysis of wormholes is given in Section 4.The Barotropic EoS is given in Section 5. The variable EoS is given in Section 6.The singular EoS is given in Section 7.A general conclusion is given in Section 8. Also adopt the units such that $c=G=1$.

\section{The Darmois - Israel Formalism}

Consider two distinct spacetime manifolds $M_{+}$and $M_{-}$ 
with metrics given by $g_{\mu \nu}^{+}\left(x_{+}^{\mu}\right)$ and $S^{i j} \bar{K}_{i j}=\left[-T_{\mu \nu} n^{\mu} n^{v}-\frac{\Lambda}{8 \pi}\right]_{-}^{+}$, in terms of independently defined coordinate systems $x_{ \pm}^{\mu}$. The manifolds are bounded by hypersurfaces $\Sigma_{+}$and $\Sigma_{-}$, respectively, with induced metrics $g_{i j}^{ \pm}$. The hypersurfaces are isometric, i.e. $g_{i j}^{+}(\xi)=g_{i j}^{-}(\xi)=g_{i j}(\xi)$, in terms of the intrinsic coordinates, invariant under the isometry. A single manifold $M$ is obtained by gluing together $M_{+}$and $M_{-}$at their boundaries, i.e. $M=M_{+} \cup M_{-}$, with the natural identification of the boundaries $\Sigma=\Sigma_{+}=\Sigma_{-}$. The second fundamental forms (extrinsic curvature) associated with the two sides of the shell are:

$$
K_{i j}^{ \pm}=-n_{\gamma}^{ \pm}\left(\frac{\partial^{2} x^{\gamma}}{\partial \xi^{i} \partial \xi^{j}}+\Gamma_{\alpha \beta}^{\gamma} \frac{\partial x^{\alpha}}{\partial \xi^{i}} \frac{\partial x^{\beta}}{\partial \xi^{j}}\right)_{\Sigma}
$$

where $n_{\gamma}^{ \pm}$are the unit normal 4-vector to $\Sigma$ in $M$, with $n_{\mu} n^{\mu}=1$ and $n_{\mu} e_{i}^{\mu}=0$.The Israel formalism requires that the normal point from $M_{-}$to $M_{+}$.For the case of a thin shell $K_{i j}$ is not continuous across $\Sigma$, so that, the discontinuity in the second fundamental form is defined as $\left[K_{i j}\right]=K_{i j}^{+}-K_{i j}^{-}$. The Einstein equation determines the relations between the extrinsic curvature and the three dimensional intrinsic energy momentum tensor are given by The Lanczos equations,

$$
S_{i j}=\frac{-1}{8 \pi}\left(\left[K_{i j}\right]-[K] g_{i j}\right)
$$

where $[K]$ is the trace of $\left[K_{i j}\right]$ and $S_{i j}$ is the surface stress-energy tensor on $\Sigma$. The first contracted GaussKodazzi equation or the "Hamiltonian" constraint

$$
G_{\mu \nu} n^{\mu} n^{\nu}=\frac{1}{2}\left(K^{2}-K_{i j} K^{i j}-{ }^{3} R\right),
$$

with the Einstein equations provide the evolution identity

$$
S^{i j} \bar{K}_{i j}=\left[-T_{\mu \nu} n^{\mu} n^{v}-\frac{\Lambda}{8 \pi}\right]_{-}^{+} .
$$

The convention $[X]=X^{+}-X^{-}$, and $\bar{X}=\frac{1}{2}\left(X^{+}+X^{-}\right)$, is used. The second contracted Gauss- Kodazzi equation or the "ADM" constraint,

$$
G_{\mu v} e_{i}^{\mu} n^{v}=K_{i ; j}^{j}-K_{, i}
$$

With the Lanczos equations gives the conservation identity

$$
S_{j ; i}^{i}=\left[T_{\mu \nu} e_{i}^{\mu} n^{v}\right]_{-}^{+} \text {. }
$$

The surface stress-energy tensor may be written in terms of the surface energy density $\sigma$, and surface pressure $p$ : $S_{j}^{i}=\operatorname{diag} \cdot(-\sigma, p, p)$. For spherically symmetric thin shell, the Lanczos equations reduce to

$$
\begin{gathered}
\sigma=\frac{-1}{2 \pi}\left[K_{\theta}^{\theta}\right] \\
p=\frac{1}{4 \pi}\left(\left[K_{\tau}^{\tau}\right]+\left[K_{\theta}^{\theta}\right]\right) .
\end{gathered}
$$

If the surface stress-energy terms are zero, the junction is denoted as a boundary surface. If surface stress terms are present, the junction is called a thin shell.

\section{Dynamics of RN Thin Shell Wormholes}

The matching of two Reissner Nordstrom space-times of $M^{ \pm}$, given by the following line elements:

$$
d s_{ \pm}^{2}=-F_{ \pm}(r) d t^{2}+F_{ \pm}^{-1}(r) d r^{2}+r^{2}\left(d \theta^{2}+\sin ^{2} \theta d \varphi^{2}\right)
$$

with

$$
F_{ \pm}=1-\frac{2 m_{ \pm}}{r}+\frac{q_{ \pm}^{2}}{r^{2}}
$$

Where $m_{ \pm}$and $q_{ \pm}$are the gravitational mass and the charge outside and inside the shell. The suffix ' + ' denotes a quantity evaluated just outside the shell and '- 'just inside the shell. Let the equation of the shell be $r_{ \pm}=R_{ \pm}(\tau)$, the history of the shell is described by the hypersurface $x_{ \pm}^{\alpha}=x_{ \pm}^{\alpha}(\tau, \theta, \varphi)$, in the regions $M^{ \pm}$, respectively; the function $R(\tau)$ describes the time evolution of the shell. The non-trivial components of the extrinsic curvature are given by

$$
\begin{gathered}
K_{\theta}^{\theta \pm}=K_{\varphi}^{\varphi \pm}=\frac{1}{R} \sqrt{F_{ \pm}+\dot{R}^{2}} \\
K_{\tau}^{\tau \pm}=\frac{1}{\sqrt{F_{ \pm}+\dot{R}^{2}}}\left(\frac{m_{ \pm}}{R^{2}}-\frac{Q^{2}}{R^{3}}+\ddot{R}\right)
\end{gathered}
$$

Note that $\dot{R}=d R / d \tau$, where the parameter $\tau$ measures proper time along the wormhole throat. From $(7,8)$ and $(10,11)$, the Lanczos equations are given by

$$
\begin{array}{r}
\sigma=\frac{-1}{2 \pi R}\left[\sqrt{F_{ \pm}+\dot{R}^{2}}\right] \\
p=\frac{1}{4 \pi R}\left[\frac{1-\frac{m_{ \pm}}{R}+\dot{R}^{2}+R \ddot{R}}{\sqrt{F_{ \pm}+\dot{R}^{2}}}\right]
\end{array}
$$

Therefore, the energy conservation can be written in the form: 


$$
\dot{\sigma}=\frac{-2 \dot{R}}{R}(\sigma+p)
$$

In this equation, the first term corresponds to a change in the throat's internal energy, while the second term corresponds to the work done by the throat's internal forces. Rearranging equation (12) to get the equation of motion of thin shell wormhole,

$$
\sqrt{F_{-}+\dot{R}^{2}}-\sqrt{F_{+}+\dot{R}^{2}}=\frac{M}{R}
$$

where $M=\sigma A$ is the rest mass of the shell, $\left(A=4 \pi R^{2}\right)$.

This equation can be written in the form

$$
\dot{R}^{2}+V(R)=0
$$

where

$$
V(R)=\frac{-M^{2}}{4 R^{2}}+\frac{1}{2}\left(F_{-}+F_{+}\right)-\frac{R^{2}}{4 M^{2}}\left(F_{-}-F_{+}\right)^{2}
$$

is the effective potential. This dynamical equation completely determines the motion of the wormhole throat.

\section{Stability Analysis}

From (14), with $p=p(\sigma)$, the conservation equation is

$$
\int \frac{d R}{R}=-\frac{1}{2} \int \frac{d \sigma}{\sigma+p(\sigma)}
$$

This relationship may then be formally inverted to: $p=p(R)$. Then, the dynamical equation (15) can be written in the form

$$
\dot{R}^{2}+F_{ \pm}-(2 \pi R \sigma)^{2}=0
$$

Where

$$
V(R)=F_{ \pm}-(2 \pi R \sigma)^{2}
$$

In the case of the static solution where, $\dot{R}=\ddot{R}=0$, and the characteristic constantsare $\sigma_{\circ}, R_{\circ}$, and $p_{\circ}$, the equations (12, 13) become:

$$
\begin{gathered}
\sigma_{\circ}=\frac{-1}{2 \pi R_{\circ}} \sqrt{F_{\circ \pm}} \\
p_{\circ}=\frac{1}{4 \pi R_{\circ}} \frac{1-m_{ \pm} / R_{\circ}}{\sqrt{F_{\text {。 }}}}
\end{gathered}
$$

By inserting the above equations $(21,22)$ into the dynamical equation, and expanding $\mathrm{V}(\mathrm{R})$ to the second order in $\left(R-R_{\circ}\right)$ lead to:

$$
V(R)=V\left(R_{\mathrm{o}}\right)+V^{\prime}\left(R_{\mathrm{o}}\right)\left(R-R_{\mathrm{o}}\right)+\frac{1}{2} V^{\prime \prime}\left(R-R_{\mathrm{o}}\right)^{2}+O(3)
$$

here prime denotes $d / d R$. In the static solution, $R=R_{\circ}$, $V\left(R_{\mathrm{o}}\right)=0$ and $V^{\prime}\left(R_{\mathrm{o}}\right)=0$, then,

$$
V(R)=1 / 2 V^{\prime \prime}\left(R_{\circ}\right)\left[R-R_{\circ}\right]^{2}
$$

The equilibrium configurations satisfying when $V^{\prime \prime}\left(R_{\mathrm{o}}\right) \succ 0$. Therefore, if the perturbation is sufficiently small, both the sign of $V^{\prime \prime}\left(R_{\circ}\right)$ and the approximately parabolic shape of the potential remain unchanged after perturbation. Under these conditions, the deformed potential gets two separate zeros within a sufficiently small neighborhood of $\mathrm{R}_{0}$. If these zeros are located at $\mathrm{R}=\mathrm{R}_{1}$ and $\mathrm{R}=\mathrm{R}_{2}\left(\mathrm{R}_{1}<\mathrm{R}_{2}\right)$, the perturbed potential is negative definite in the interval $\left(\mathrm{R}_{1} ; \mathrm{R}_{2}\right)$. Therefore, the assumption $V^{\prime \prime}\left(R_{\circ}\right) \succ 0$ entails oscillations between the slightly separated turning points $\mathrm{R}_{1}$ and $\mathrm{R}_{2}$ when the perturbation is sufficiently small. This situation describes linearized thin shell stability against radial perturbations. On the contrary, the case $V^{\prime \prime}\left(R_{\circ}\right) \prec 0$ implies instability. The truncated expansion (24) is essential to the linearized stability analysis considered here. The present approach is not conclusive when applied to throats in static equilibrium with vanishing $V^{\prime \prime}\left(R_{\circ}\right)$.

\section{Barotropic Equation of State}

The linear barotropic equation of state (EoS),

$$
p=\omega \sigma
$$

Differentiating the conservation equation (18) led to:

$$
R \frac{d \sigma}{d R}=-2(\sigma+p)
$$

From equations $(25,26)$, the local solution will be

$$
\sigma(R)=\sigma_{\circ}\left(\frac{R_{\circ}}{R}\right)^{2(\omega+1)}
$$

which satisfies the condition $\sigma\left(R_{\circ}\right)=\sigma_{\circ}$. Combining this result with equation (20) will led to the corresponding effective potential:

$$
V(R)=F_{ \pm}-4 \pi^{2} R^{2} \sigma_{\circ}^{2}\left(\frac{R_{\circ}}{R}\right)^{4(\omega+1)}
$$

Using (21) the condition $V\left(R_{\mathrm{o}}\right)=0$, is automatically satisfied. However the first derivative of $\mathrm{V}(\mathrm{R})$ evaluated at $\mathrm{R}$ $=\mathrm{R}_{0}$ vanishes if and only if

$$
\omega=\frac{-\left(1-m_{ \pm} / R_{\circ}\right)}{2 F_{\mathrm{o}}}
$$

From (27), the local solution becomes constant when 
$\omega=-1$ and equation (29) occurs only at $R_{\mathrm{o}}=3 m_{ \pm}$, and $q=0$. From (21) the corresponding (local) energy density function, is

$$
\sigma(R)=\left.\sigma_{\circ}\right|_{R_{o}=3 m_{ \pm}}=\frac{-\sqrt{3 m_{ \pm}^{2}+q^{2}}}{18 \pi m_{ \pm}^{2}}
$$

By doing the second derivative of equation (26) I obtain,

$$
(R \sigma)^{\prime \prime}=\frac{2}{R}(1+2 \partial p / \partial \sigma)(\sigma+p)
$$

Now define a parameter $\gamma$ by the relation

$$
\gamma^{2}(\sigma)=\partial p / \partial \sigma
$$

Note that, $\gamma$ is a useful parameter related to the equation of state. Differentiate equation (20) to get,

$$
V^{\prime}(R)=F_{ \pm}^{\prime}+8 \pi^{2} R \sigma(\sigma+2 p)
$$

and

$$
V^{\prime \prime}(R)=F_{ \pm}^{\prime \prime}-8 \pi^{2}\left\{(\sigma+2 p)^{2}+2 \sigma(\sigma+p)\left(1+2 \gamma^{2}\right)\right\}
$$

Evaluating this equation at the static solution,

$$
\begin{aligned}
& V^{\prime \prime}\left(R_{\circ}\right)=F_{\circ \pm}^{\prime \prime}-\frac{2}{R_{\circ}^{2}}\left\{F_{\circ \pm}^{-1}\left(1-\frac{m_{ \pm}}{R_{\circ}}-F_{\circ \pm}\right)^{2}\right. \\
& \left.-\left(1+2 \gamma_{\circ}^{2}\right)\left(1-\frac{m_{ \pm}}{R_{\circ}}-2 F_{\circ \pm}\right)\right\}
\end{aligned}
$$

The equation of motion for the wormhole throat, at this order of approximation, is

$$
\dot{R}^{2}=-\frac{1}{2} V^{\prime \prime}\left(R-R_{\mathrm{o}}\right)^{2}+O(3) .
$$

Thus the wormhole is stable if and only if $V^{\prime \prime}\left(R_{\mathrm{o}}\right) \succ 0$. The relation where $V^{\prime \prime}\left(R_{\mathrm{o}}\right)=0$ corresponding to:

$$
\begin{aligned}
& V^{\prime \prime}\left(R_{\circ}\right)=-\frac{2}{R_{\circ}^{2}}\left\{\frac{2 m_{ \pm}}{R_{\circ}}-\frac{3 q^{2}}{R_{\circ}^{2}}+\frac{\left(\frac{m_{ \pm}}{R_{\circ}}-\frac{q^{2}}{R_{\circ}^{2}}\right)^{2}}{\left(1-\frac{2 m_{ \pm}}{R_{\circ}}+\frac{q^{2}}{R_{\circ}^{2}}\right)}\right. \\
& \left.+\left(1+2 \gamma_{\circ}^{2}\right)\left(1-\frac{3 m_{ \pm}}{R_{\circ}}+\frac{2 q^{2}}{R_{\circ}^{2}}\right)\right\}
\end{aligned}
$$

It implies that every static throat with $\gamma_{0}^{2}$ bounded at $R_{\mathrm{o}}=3 m_{ \pm} \quad$ and $\quad q=0 \quad$ is characterized by $V^{\prime \prime}\left(R_{\circ}=3 m_{ \pm}\right)=-2 / 9 m_{ \pm}^{2}$, and $q=0$. This result precludes the existence of stability regions including the throat radius $R_{\circ}=3 m_{ \pm}$. It also imposes a clear cut separation between possible stability regions defined for $R_{\circ} \succ 3 m_{ \pm}$or $R_{\circ} \prec 3 m_{ \pm}$.

\section{Variable Equation of State}

The property $p^{\prime}=\frac{\partial p(\sigma)}{\partial \sigma} \sigma^{\prime}$, is suitable for EoS of the form $p=p(\sigma)$. The integrability of (14) with variable EoS $p=p(\sigma, R)$, will be

$$
\sigma^{\prime}=\frac{-2}{R}[\sigma+p(\sigma, R)]
$$

Therefore, the present analysis of linearized stability with variable EoS $p=p(\sigma, R)$ requires the more general expression:

$$
p^{\prime}=\frac{\partial p}{\partial \sigma} \sigma^{\prime}+\frac{\partial p}{\partial R}
$$

which takes into account the explicit dependence of pressure on throat radius. Using the new form of $p^{\prime}$ in equation (37) to get,

$$
\begin{aligned}
& V^{\prime \prime}\left(R_{\circ}\right)=-\frac{2}{R_{\circ}^{2}}\left\{\frac{2 m_{ \pm}}{R_{\circ}}-\frac{3 q^{2}}{R_{\circ}^{2}}+\frac{\left(\frac{m_{ \pm}}{R_{\circ}}-\frac{q^{2}}{R_{\circ}^{2}}\right)^{2}}{\left(1-\frac{2 m_{ \pm}}{R_{\circ}}+\frac{q^{2}}{R_{\circ}^{2}}\right)}+\right. \\
& \left.\left(1+2 \gamma_{\circ}^{2}\right)\left(1-\frac{3 m_{ \pm}}{R_{\circ}}+\frac{2 q^{2}}{R_{\circ}^{2}}\right)\right\}+8 \pi \zeta_{\circ} \sqrt{1-\frac{2 m_{ \pm}}{R_{\circ}}+\frac{q^{2}}{R_{\circ}^{2}}}
\end{aligned}
$$

where $\zeta_{0} \equiv-\left.\frac{\partial p}{\partial R}\right|_{R=R_{\text {s }}}$. Assuming that $\gamma_{0}^{2}$ is bound at $R_{\mathrm{o}}=\frac{3}{2} m_{ \pm}$and $q^{2}=\frac{9}{8} m_{ \pm}^{2}$, then

$$
V^{\prime \prime}\left(R_{\mathrm{o}}=\frac{3}{2} m_{ \pm}, q=\frac{3}{2 \sqrt{2}} m_{ \pm}\right)=\frac{8}{\sqrt{6}} \pi \zeta 。
$$

which depends on the selected EoS through $\zeta_{0}$.

Rewrite (25) in terms of the linear variable EoS:

$$
p=\omega(R) \sigma
$$

where $\mathrm{R}$ is the radius of thin-shell. In this mathematical modeling $\mathrm{p}$ is supposed to be:

$$
p=\frac{A}{R^{n}} \sigma
$$

where $A$ and $n$ are real constants, and $n \neq 0$. Inserting (43) into (38) to get,

$$
\frac{d}{d R} \sigma^{2}+\frac{4}{R}\left(1+\frac{A}{R^{n}}\right) \sigma^{2}=0
$$

The solution of equation (44) is 


$$
\sigma^{2}(R)=\sigma_{\circ}^{2}\left(\frac{R_{\circ}}{R}\right)^{4} \exp \left(\frac{4 A}{n}\left(\frac{1}{R^{n}}-\frac{1}{R_{\circ}^{n}}\right)\right)
$$

which satisfies the condition $\sigma^{2}(R)=\sigma_{\circ}^{2}$ at the static equilibrium radius $R=R_{\mathrm{o}}$. Using (45) and (20) to get

$$
V(R)=F_{ \pm}-4 \pi^{2} R^{2} \sigma_{\circ}^{2}\left(\frac{R_{\circ}}{R}\right)^{4} \exp \left(\frac{4 A}{n}\left(\frac{1}{R^{n}}-\frac{1}{R_{\circ}^{n}}\right)\right)
$$

Differentiating $\mathrm{V}(\mathrm{R})$ with respect to $\mathrm{R}$ and evaluating the resulting expression at $R=R_{\circ}$, I get $V^{\prime}\left(R_{\circ}\right)=0$ if and only if

$$
A=\frac{-\left(R_{\circ}^{n}-m_{ \pm} R_{\circ}^{n-1}\right)}{2 F_{\circ \pm}}
$$

Inserting (47) into (45) and evaluating at $R_{\circ}=3 m_{ \pm}$, to obtain

$$
\begin{aligned}
& \sigma^{2}(R)=\frac{3 m_{ \pm}^{2}}{4 \pi^{2} R^{4}}\left(1+\frac{q^{2}}{3 m_{ \pm}^{2}}\right) \exp \\
& \left(\frac{4}{n}\left(1-\left(\frac{3 m_{ \pm}}{R}\right)^{n}\right)\left(1+\frac{q^{2}}{3 m_{ \pm}^{2}}\right)^{-1}\right)
\end{aligned}
$$

which is not constant when $\mathrm{n} \neq 0$. The limiting behavior of equation (48) with $\mathrm{n}$ tends to 0 , or let $R=3 m_{ \pm}$, will be

$$
\sigma^{2}\left(R=3 m_{ \pm}\right)=\frac{1}{108 \pi^{2} m_{ \pm}^{2}}\left(1+\frac{q^{2}}{3 m_{ \pm}^{2}}\right)
$$

The fact that equation (48) approaches a constant function, as $\mathrm{n}$ tends to 0 is expected on the basis of (30), which is derived in the framework of linear barotropic EoS. Substituting (47) into (46), determining $V^{\prime \prime}(R)$, evaluating at $R=R_{\circ}$, and introducing the dimensionless throat radius $\chi_{\circ}=R_{\circ} / 2 m_{ \pm}$, to get

$$
\begin{aligned}
& V^{(2)}\left(\chi_{\circ}\right)=\frac{1}{\chi_{\circ}^{3}\left(\chi_{\circ}-1+q^{2} / 4 \chi_{\circ} m_{ \pm}^{2}\right)}\left[2 n \chi_{\circ}^{2}\right. \\
& \left.-\chi_{\circ}(3 n+1)+n+q^{2}\left(1-\frac{1}{4 \chi_{\circ}}+\frac{n}{2}-\frac{n}{4 \chi_{\circ}}\right) / m_{ \pm}^{2}\right]
\end{aligned}
$$

where $V^{(2)}\left(\chi_{\circ}\right) \cong 4 m_{ \pm}^{2} V^{\prime \prime}\left(R_{\circ}\right)$. The second (dimensionless) derivative of $\mathrm{V}$ takes the approximate form

$$
V^{(2)}\left(\chi_{\circ}\right) \approx \frac{-1}{\left(\chi_{\circ}-1+q^{2} / 4 \chi_{\circ} m_{ \pm}^{2}\right)}
$$

as $\chi_{\circ} \rightarrow 1$. This result guarantees the negativity of $V^{(2)}\left(\chi_{\mathrm{o}}\right)$ near $\chi_{0}=1$. Therefore, linearized stability can be achieved only if the sign of $V^{(2)}\left(\chi_{\circ}\right)$ turns positive at some $\chi_{\circ} \succ 1$. Hence the importance of classifying the roots of $V^{(2)}\left(\chi_{0}\right)=0$ for non-vanishing $\mathrm{n}$. The cubic equation,

$$
\begin{aligned}
& 2 n \chi_{\circ}^{3}-\chi_{\circ}^{2}(3 n+1)+\left(n+\frac{q^{2}}{m_{ \pm}^{2}}+\frac{n q^{2}}{2 m_{ \pm}^{2}}\right) \chi \\
& +\frac{q^{2}}{m_{ \pm}^{2}}\left(-\frac{1}{4}-\frac{n}{4}\right)=0
\end{aligned}
$$

admits the roots:

$$
\begin{aligned}
& \chi_{\circ}=\sqrt[3]{\left(\frac{-b^{3}}{27 a^{3}}+\frac{b c}{6 a^{2}}-\frac{d}{2 a}\right)+\sqrt{\left(\frac{-b^{3}}{27 a^{3}}+\frac{b c}{6 a^{2}}-\frac{d}{2 a}\right)^{2}}} \\
& +\sqrt[3]{\left(\frac{c}{3 a}-\frac{b^{2}}{9 a^{2}}\right)^{3}} \\
& +\sqrt{\left.\frac{-b^{3}}{27 a^{3}}+\frac{b c}{6 a^{2}}-\frac{d}{2 a}\right)-\sqrt{\left(\frac{-b^{3}}{27 a^{3}}+\frac{b c}{6 a^{2}}-\frac{d}{2 a}\right)^{2}}}-\frac{b}{3 a}
\end{aligned}
$$

where $a=2 n, b=-(3 n+1), c=n+\frac{q^{2}}{m_{ \pm}^{2}}+\frac{n q^{2}}{2 m_{ \pm}^{2}} \quad, \quad$ and $d=\frac{q^{2}}{m_{ \pm}^{2}}\left(-\frac{1}{4}-\frac{n}{4}\right)$.Equations (42) and (43) completely remove the special character of throat radius $R_{\circ}=3 m_{ \pm},\left(\chi_{\circ}=3 / 2\right)$ which arises in the barotropic case. Using (50) to determine $V^{(2)}(3 / 2)$ as a function of $\mathrm{n}$, then

$$
V^{(2)}\left(\frac{3}{2}\right)=\frac{16\left[n-\frac{3}{2}+q^{2}\left(n+\frac{5}{2}\right) / 3 m_{ \pm}^{2}\right]}{27\left(1+q^{2} / 3 m_{ \pm}^{2}\right)}
$$

Therefore $V^{(2)}(3 / 2)$ is positive whenever $n \succ 3 / 2$, so static throats with dimensionless radius $\chi_{\circ}=3 / 2$ can be linearly stable.

\section{Singular Equation of State}

In the cases of barotropic EoS (25) and variable EoS (43), it is found that the second and higher derivatives of $\mathrm{V}$ diverge as the static equilibrium radius approaches $2 m_{ \pm}$and $\mathrm{q}=0$. It is worth investigating if some of these irregularities can be mitigated by choosing a different type of variable EoS (i.e. a linear EoS with singular coefficient). In this limit the pressure-density ratio takes the approximate form

$$
\frac{p_{\circ}}{\sigma_{\circ}}=\frac{-\left(R_{\circ}-m_{ \pm}\right)}{2 R_{\circ} F_{\circ \pm}}
$$

which is unbounded. This result is totally independent of the selected EoS. The above limit expression is compatible with the choice

$$
p=\frac{\psi}{F_{ \pm}} \sigma
$$


which is another particular case of (42). The constant parameter $\psi$ is to be determined at each static equilibrium radius. This EoS implies that, the dynamical pressure-density ratio $p / \sigma$ becomes unbounded as $\mathrm{R}$ approaches $2 m_{ \pm}$and $\mathrm{q}$ $=0$. Also, equation (56) takes the approximate form $p \approx \psi \sigma$ when $R \succ \succ 2 m_{ \pm}$and $\mathrm{q}=0$. The simple dependence of $\mathrm{p}$ on $\mathrm{R}$ and $\sigma$ displayed in (56), as well as the associated limiting behaviors make this EoS an interesting candidate for linearized stability analysis. Evaluating (56) at $R=R_{\text {。 }}$ and combining the result with the general expression for $p_{\circ} / \sigma_{\circ}$, derived from (21) and (22), to get

$$
\psi=\frac{-\left(R_{\circ}-m_{ \pm}\right)}{2 R_{\circ}}
$$

which is negative definite when $R_{\circ} \succ 2 m_{ \pm}$. It tends to $-1 / 4$ as $R_{\circ} \rightarrow 2 m_{ \pm}$, and takes the approximate form $\psi=-1 / 2$ when $R \succ \succ 2 m_{ \pm}$. The fact that the equilibrium values of $\omega$ and $\psi$ tend to the same limit as $\mathrm{R}_{0}$ becomes arbitrarily large motivates a comparison of linearzed stability properties of (25) and (56). The following analysis shows that the approximate identification of these EoS for $R_{\circ} \succ \succ 2 m_{ \pm}$is misleading, as the variability of (56) dramatically modifies the values of $V^{\prime \prime}\left(R_{\mathrm{o}}\right)$ in the whole interval $\left(2 m_{ \pm}, \infty\right)$. Inserting (56) into (38) and find the differential equation,

$$
\frac{d}{d R} \sigma^{2}=\frac{-4}{R}\left(1+\frac{\psi}{F_{ \pm}}\right) \sigma^{2}
$$

which admits the local solution

$$
\begin{aligned}
& \sigma^{2}=\sigma_{\circ}^{2}\left(\frac{R_{\circ}}{R}\right)^{4}\left(\frac{R_{\circ}^{2}-2 m_{ \pm} R_{\circ}+q^{2}}{R^{2}-2 m_{ \pm} R+q^{2}}\right)^{2 \psi} \\
& \exp \left(\frac{4 m_{ \pm} \psi\left(R-R_{\circ}\right)}{m_{ \pm}^{2}-q^{2}}\right)
\end{aligned}
$$

The above expression fulfills the condition $\sigma^{2}=\sigma_{\circ}^{2}$ at the equilibrium radius $R=R_{\circ}$. Using (59) and (20) to get

$$
\begin{aligned}
& V(R)=F_{ \pm}-4 \pi^{2} R^{2} \sigma_{\circ}^{2}\left(\frac{R_{\circ}}{R}\right)^{4}\left(\frac{R_{\circ}^{2}-2 m_{ \pm} R_{\circ}+q^{2}}{R^{2}-2 m_{ \pm} R+q^{2}}\right)^{2 \psi} \\
& \exp \left(\frac{4 m_{ \pm} \psi\left(R-R_{\circ}\right)}{m_{ \pm}^{2}-q^{2}}\right)
\end{aligned}
$$

Combined with (21), this expression satisfies the equilibrium condition $V\left(R_{\circ}\right)=0$, also $V^{\prime}\left(R_{\circ}\right)$ vanishes if and only if $\psi$ is given by (57). Differentiate equation (58) twice times to get:

$$
V^{\prime \prime}\left(R_{\circ}\right)=2 m_{ \pm} / R_{\circ}^{3}
$$

with $q=0$, this is positive definite on the interval $\left(2 m_{ \pm}, \infty\right)$ and remains finite as the throat equilibrium radius
$\mathrm{R}_{0}$ approaches the Schwarzschild radius. The values of the higher derivatives of (60) at $R=R_{\circ}$, can also be determined. These quantities become unbounded as $R_{\circ} \rightarrow 2 m_{ \pm}$.

\section{Conclusions}

In the framework of Darmois-Israel formalism, the charged thin shell wormholes with variable equations of state are constructed, by matching the external RN solution with the internal RN solution across the singular surface. The linearized stability analyses of spherically-symmetric thin-shell wormholes by considering linearized radial perturbations around some assumed static solution of the Einstein field equations are obtained.

Introduce two types of variable EoS leading to linearly stable RN thin-shell wormholes were proposed. In the case of EoS (43) I find semi-infinite stability regions determined by positive values of $V^{(2)}\left(\chi_{0}\right)$. The corresponding boundaries get arbitrarily close to the horizon $\left(\chi_{\circ}=1\right)$ as $n \rightarrow \infty$. It turns out that $V^{(2)}$ and the higher derivatives of $\mathrm{V}$ evaluated at $\chi$ 。 become unbounded as $\chi_{\circ} \rightarrow 1$. The case of EoS (56) is substantially different since $V^{\prime \prime}\left(R_{\mathrm{o}}\right)$ is definite positive throughout the entire interval $\left(2 m_{ \pm}, \infty\right)$, and remains bounded as $R_{\circ} \rightarrow 2 m_{ \pm}$. However, the higher derivatives of $\mathrm{V}$ also diverge as $R_{\circ} \rightarrow 2 m_{ \pm}$. Remarkably, linearized stability criteria [14], have been used despite the divergent derivatives of $\mathrm{V}$ as $R_{\circ} \rightarrow 2 m_{ \pm}$.

The parameter $\gamma_{0}^{2}$, squared sound speed, defined in (32), is negative definite for linearly stable throats with radii $R_{\circ} \succ 3 m_{ \pm}[15]$.

It is not clear whether thin shell EoS arising from modified relativistic gas models would be compatible with the linear form (42) in certain limiting situations. Also, the motivation for including the singular coefficient in (56) may be questioned [16]. However, the emergence of linearized stability in the context of RN thin shell wormholes makes (42) an interesting choice. Particularly, the achieved stabilization at every throat radius is a remarkable consequence of (56) which deserves further attention.

In the first case of variable EoS, with regular coefficients, a sequence of semi-infinite stability regions is found such that every throat in equilibrium becomes stable for a particular subsequence. In the second case, a singular EoS (in such variable EoS the coefficients is explicitly dependent on throat radius), the second derivative of the effective potential is positive definite, so linearized stability is guaranteed for every equilibrium radius.

Finally, these variable EoS could have a significant impact on the stability of other gravitational sources incorporating thin shells (for example, lower-dimensional, cylindrical, intra-galactic, higher-dimensional, rotating, and 
Einstein-Maxwell-Gauss- Bonnet wormholes, as well as stars and circumstellar shells, gravastars, and multi-layer spherical systems).

\section{References}

[1] W. Israel, Nuovo Cimento B, 44, 1, 1966.

[2] K. Kuchar, Czech J. Phys. B, 18, 435, 1968.

[3] V.A. Berezin, V.A. Kuzmin and I.I. tkachev, Phys. Rev. D., 36 , $2919,1987$.

[4] H. Sato, Prog. Theor. Phys., 80, 96, 1988.

[5] S. W. Kim, Phys. Lett. A, 166, 13, 1992.

[6] F.S.N. Lobo and P. Crawford, Class. Quantum Grav., 22, 4869, 2005.
[7] M. Visser, Lorentzian Wormholes, AIP Press, New York, 1996.

[8] E.F. Eiroa and C. Simeone, Phys. Rev. D., 70, 044008, 2004.

[9] N. M. Garcia, F.S.N. Lobo and M. Visser, Phys. Rev. D, 86, 044026, 2012.

[10] J. Ponce de Leon, Class. Quant. Grav., 29, 135009, 2012.

[11] F. Rahaman, M. Kalam and S. Chakraborty, Acta Phys. Polon. B, 40, 25, 2009.

[12] P. K. F. Kuhfittig, arXiv: gr-qc: 0707.4665.

[13] K. A. Bronnikov, L. N. Lipatova, I. D. Novikov and A. A. Shatskiy, Grav. Cosmol., 19, 269, 2013.

[14] P. K. F. Kuhfittig, Acta Phys. Polon. B, 41, 2017, 2010.

[15] A. Eid, New Astro., 39, 72, 2015.

[16] E. Mottola, Acta Phys. Polon. B, 41, 2031, 2010. 